\title{
РАБОТА, ОБРАЗОВАНИЕ И ГРАМОТНОСТЬ В РОССИИ: ПРОБЛЕМА НЕКОНСИСТЕНТНОСТИ
}

Статья посвящена исследованию проблемы неконсистентности (несоответствия) в сфере образования и труда. Мы используем результаты международного исследования компетентности взрослых (PIAAC), в рамках которого на репрезентативной для страны выборке были измерены грамотность чтения и математическая грамотность людей трудоспособного возраста. Тестовые результаты в странах ОЭСР демонстрируют связь с уровнем формального образования, однако в России мы наблюдаем несколько отклонений. Анализ позволил выявить три вида неконсистентности. Мы обнаружили, что в России не наблюдается линейной зависимости между уровнем формального образования и измеренной грамотностью. Прирост компетентности замедляется на уровне высшего образования, тогда как на нижних образовательных уровнях на фоне ведущих мировых стран - падения грамотности не наблюдается. При сравнении России с развитыми странами выяснилось, что большая доля людей с низким уровнем грамотности занимает высококвалифицированные рабочие места. При оценке распределения людей с тем или иным формальным образованием на рынке труда в России мы видим картину весьма близкую к той, что наблюдается в странах ОЭСР. По всей видимости, эта неконсистентность была скрыта из-за недостаточной надежности для нашей страны основного (а в большинстве случаев единственного) и для социологических, и для экономических исследований образовательного индикатора: наличия диплома об образовании. Третий эмпирический результат демонстрирует соотношение важности

Дмитрий Сергеевич Попов - к.соц.н., старший научный сотрудник Национального исследовательского университета «Высшая школа экономики», Москва, Россия.Электронная почта: dmitry_popov@sociolog.net

Анна Владимировна Стрельникова - к.соц.н., доцент Национального исследовательского университета «Высшая школа экономики», доцент ГАУГН, Москва, Россия. Электронная почта: astrelnikova@hse.ru 
измеренной грамотности и наличия диплома при трудоустройстве. В нашей стране оба фактора оказываются значимыми, но измеренная грамотность сильнее связана с шансом быть трудоустроенным по сравнению с формальным дипломом. При этом различие по уровню доходов между респондентами с низким и высоким уровнем грамотности в России значительно меньше, чем в странах ОЭСР. Интерпретация результатов количественных измерений реализована с привлечением качественных данных проекта по исследованию жизненных траекторий.

Ключевые слова: социальное неравенство, образование, неконсистентность в образовании, грамотность, компетентностный подход

DOI: $10.17323 / 727-0634-2017-15-2-267-280$

На фоне доступности высшего образования в России образовательные программы становятся неоднородными по качеству (Селезнева 2003: 42-47; Карпенко 2012:9-62). Это порождает противоречия не только между квалификацией специалистов и реальным спросом на рынке труда, но и между образованием и образованностью (грамотностью). Подобные противоречия приводят к получению парадоксальных исследовательских результатов. Например, более 80 \% выпускников вузов работают не по специальности, однако при этом формальное наличие дипломов о высшем образовании приводит к повышению уровня оплаты труда (Гимпельсон и др. 2009). И наоборот, люди с высоким измеренным уровнем грамотности могут обладать низким уровнем формального образования и заработной платы (Кузьмина, Попов 2015). В этой статье мы обращаемся к проблеме неконсистентности несоответствия позиции на рынке труда и показателей в области образования, рассматриваемого и как формальный образовательный статус, и как измеренный достигнутый уровень грамотности. Отметим, что неконсистентность неизбежно присутствует в любом обществе, однако значительный ее масштаб - это свидетельство дерегуляции и симптом кризисных явлений.

В социологической теории предлагается как минимум две перспективы рассматриваемой проблемы, которые вслед за Джоном Мейером можно обозначить как «социализационную» и «аллокативную» (Meyer 1977). «Социализационный» подход, укорененный в функционализме, предполагает, что получение знаний и увеличение численности образованных людей приводит к усложнению общества и развитию социальных институтов. Во многом на основе этой идеи возникают и развиваются крупные международные исследования грамотности и навыков, такие, как программа PIAAC (OECD 2013), материалы которой используются в статье. На этот подход опираются и неоинституциалистские исследования в образовании (см., напр., Powell, DiMaggio 1991; Wiseman et al. 2014). В логике данного подхода эффективность института образования определяется приростом знаний. Соответственно, можно ожидать линейной зависимости между количеством потраченных на образование лет (или уровнем формального 
диплома) и объемом полученных знаний. Если же такой зависимости не наблюдается, впору говорить об институциальном нарушении, которому исследователи должны уделить пристальное внимание.

«Аллокативная» перспектива, связанная с критической теорией в социологии, показывает, что образование связано со структурой общества и «распределяет» людей в нем (Collins 1979; Бурдье, Пассрон 2007). Результат образования при этом является авторитетным знанием (authoritative knowledge), т.е. формальными навыками, открывающими доступ в определенные социальные сегменты. Эффективность такого распределения и воспроизводства общества зависит и от процедур формальной сертификации (присвоения дипломов), и от знаний, которые образование способно транслировать. В ситуации, когда формальные дипломы оказываются неподкрепленными знаниями, приходится ставить под сомнение эффективность формального образования как аллокативной системы. В этом случае, вероятно, в дело вступают альтернативные механизмы распределения.

Таким образом, выявление неконсистентности в рассматриваемой сфере может стать серьезным аргументом в пользу необходимости регулирования социальной политики в сфере образования и дополнительной профессиональной подготовки. Поэтому мы предлагаем рассмотреть, насколько формальный индикатор, при помощи которого принято определять уровень образования (наличие диплома), соответствует измеренным уровням знаний и компетентности россиян. Это один из способов определения качества и стабильности результатов образования на национальном уровне. Интерес представляет не только рассогласованность формальных индикаторов, но также «содержательная» неконсистентность, которая скрывается за этими индикаторами. Можно предположить, что наличие неконсистентности в области образования будет иметь последствия в сфере труда. Поэтому важно изучать вопрос о связи между уровнем образования, измеренной компетентности и типом занятости (в логике Международной стандартной классификации занятий ООН).

Другой проблемой в области занятости является востребованность формального образования (диплома) и реальной компетентности выпускников. В России уже имеются масштабные исследования, посвященные отдаче от образования на рынке труда или «эффективности» образования (Лукьянова 2010; Рощин, Рудаков 2015). Однако в этих работах используется лишь один показатель (наличие формального диплома). Использование единственного индикатора с одной стороны привлекательно, поскольку во многих случаях приводит к сопоставимости результатов, причем как внутристрановых, так и международных. Вместе с тем надежность этого индикатора при измерении образовательного уровня в нашей стране вызывает сомнение.

Таким образом, содержательная задача нашей статьи состоит в анализе ситуаций неконсистентности (или, напротив, кристаллизации) в пространствах образования и труда, а при их выявлении - в объяснении их причин 
и оценке последствий для российского общества. Наряду с этим существует и методическая задача, которая связана с необходимостью оценки эффективности основного показателя уровня образования - наличия диплома об образовании. Оценка возможна на разных уровнях образования благодаря наличию тестовых показателей, а также возможности их сопоставления с аналогичными данными в других странах.

\section{Используемые данные и методы анализа}

Мы опираемся на данные «Программы международной оценки компетентности взрослых» (Programme for the International Assessment of Adult Competences, PIAAC). Программа реализуется в десятках ведущих стран мира, включая Россию (OECD 2013). Проект PIAAC стал первым опытом измерения грамотности взрослых в России, первая волна проекта была завершена в 2013 г. Он ориентирован на изучение компетентности взрослого населения в возрасте 16-65 лет, количество респондентов в России - 3892 чел. В рамках проекта используется биографический блок вопросов, а также блок специальных тестовых инструментов. Методы работы с количественными данными включали в себя проведение одномерного и двумерного анализа: группировка данных, расчет частот и процентилей, статистическое сравнение средних значений, дисперсионный анализ с расчетом апостериорных критериев, расчет таблиц сопряженности.

Как дополнительный источник при интерпретации количественных результатов используются качественные данные проекта «Межпоколенная социальная мобильность от XX века к XXI: четыре генерации российской истории» под руководством Зинаиды Голенковой, нацеленного на изучение жизненных траекторий россиян в нескольких измерениях: занятость, образование, уровень жизни, субъективные оценки своего жизненного пути. ${ }^{1}$

\section{Результаты: выявленная неконсистентность в сфере образования и труда}

Грамотность чтения и математическая грамотность - важнейшие аналитические индикаторы, которые в странах ОЭСР линейно связаны с уровнем образования (OECD 2013) и вполне надежно предсказывают отдачу на рынке труда (Hanushek et al. 2015). Однако анализ российских данных международной программы $P I A A C$ позволяет говорить о наличии сразу нескольких рассогласованностей (неконсистентностей) в области образования и труда в нашей стране. Для анализа мы используем как

\footnotetext{
В рамках статьи используются материалы качественного этапа (85 интервью, собранных в 2015 г.).
} 
классический индикатор - наличие диплома о формальном образовании, так и измеренную компетентность в области математики и чтения.

Таблий 1.

Взаимосвязь средних значений грамотности и уровня образования*

\begin{tabular}{|c|c|c|c|c|c|}
\hline & \multicolumn{2}{|c|}{ Россия } & \multicolumn{3}{|c|}{ Страны ОЭСР } \\
\hline & 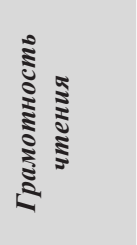 & 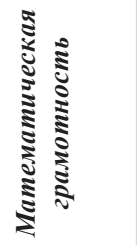 & 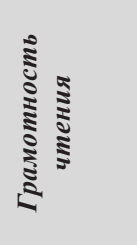 & 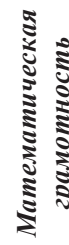 & \\
\hline Уровень образования & \multicolumn{5}{|c|}{$\begin{array}{c}\text { Средний тестовый балл } \\
\text { (в скобках указано стандартное отклонение) }\end{array}$} \\
\hline $\begin{array}{l}\text { Низкий - } \\
\text { (ниже среднего, неполное } \\
\text { среднее) }\end{array}$ & $\begin{array}{l}259 \\
(42)\end{array}$ & $\begin{array}{l}250 \\
(45)\end{array}$ & $\begin{array}{l}241 \\
(47)\end{array}$ & & $\begin{array}{l}231 \\
(51)\end{array}$ \\
\hline $\begin{array}{l}\text { Средний- } \\
\text { (общее среднее, средне- } \\
\text { специальное) }\end{array}$ & $\begin{array}{l}278 \\
(39)\end{array}$ & $\begin{array}{l}274 \\
(38)\end{array}$ & $\begin{array}{l}270 \\
(39)\end{array}$ & & $\begin{array}{l}265 \\
(43)\end{array}$ \\
\hline $\begin{array}{l}\text { Высокий-(высшее, } \\
\text { несколько высших) }\end{array}$ & $\begin{array}{l}282 \\
(38)\end{array}$ & $\begin{array}{l}277 \\
(36)\end{array}$ & $\begin{array}{l}293 \\
(38)\end{array}$ & & $\begin{array}{l}289 \\
(42)\end{array}$ \\
\hline
\end{tabular}

\section{F-статистика}

\begin{tabular}{lrrrr} 
Результат дисперсионного & \multicolumn{4}{c}{ (в скобках указан уровень значимости) } \\
\cline { 2 - 5 } анализа & 48,4 & 66,1 & 15472,4 & 16206,7 \\
& $(0,000)$ & $(0,000)$ & $(0,000)$ & $(0,000)$ \\
\hline
\end{tabular}

Множественные сравнения Разность средних тестовых баллов для различных (критерий Шеффе) уровней образования (в скобках указана стандартная оцибка и уровень значимости)

\begin{tabular}{lrrrr}
\hline Высокий - низкий & $23,3(2,4 ;$ & $26,5(2,3 ;$ & $51,7(0,3 ;$ & $58,6(0,3 ;$ \\
& $0,000)$ & $0,000)$ & $0,000)$ & $0,000)$ \\
\hline Высокий - средний & $4,5(1,6 ;$ & $2,4(1,5 ;$ & $22,8(0,2 ;$ & $24,1(0,3 ;$ \\
& $0,020)$ & $0,285)$ & $0,000)$ & $0,000)$ \\
\hline Средний - низкий & $18,8(2,7 ;$ & $24,0(2,6 ;$ & $28,9(0,3 ;$ & $34,5(0,3 ;$ \\
& $0,000)$ & $0,000)$ & $0,000)$ & $0,000)$ \\
\hline
\end{tabular}

* Все различия и для России, и для ОЭСР значимы на уровне 0,05 , за исключением различий в средних баллах математической грамотности между группами высокого и среднего образования в России. Также на уровне 0,01 между этими же российскими группами перестает быть значимым и различие в средних баллах грамотности чтения. 
Изучая взаимосвязь формального образования и измеренного уровня компетентности, мы выявили первую рассогласованность, связанную с тем, что россияне без высшего образования показали достаточно высокие результаты. При этом обладающие различными уровнями формального образования, имеют низкую межгрупповую вариацию результатов тестов грамотности, т.е. компетенции россиян «неустойчивы» относительно той или иной образовательной группы (табл. 1). Получение диплома о высшем образовании в России не означает автоматического прироста компетентности. Это ставит под сомнение надежность формального диплома как основного образовательного индикатора.

Дисперсионный анализ показал, что как в России, так и в странах ОЭСР уровень формального образования является значимым фактором, влияющим на различие в средних баллах по измеренным компетенциям в чтении и математике (статистика Фишера во всех случаях значима на уровне 0,000$)$. Россияне, имеющие средний уровень образования (общее среднее, средне-специальное), демонстрируют практически те же тестовые баллы, что и их более образованные сограждане, обладающие дипломами о высшем образовании. Дальнейший анализ этого парадокса при помощи расчета критерия Шеффе показал, что в России не для всех образовательных групп можно говорить о различиях в средних тестовых баллах, обусловленных образованием. Различие в средних баллах математической грамотности между россиянами со средним и высоким уровнем образования составило всего 2,4 балла и оказалось незначимым на уровне 0,05 , а грамотности чтения - на уровне 0,01 . Это означает, что формальный высокий образовательный статус (в данном случае - обладание дипломом о высшем образовании) не гарантирует прироста компетенций у его обладателя по сравнению с теми, кто имеет среднее и среднее специальное образование.

Результаты анализа разности средних тестовых баллов для различных уровней образования показывают, что соответствие между шкалой компетенций и шкалой образования воспроизводится в полученных результатах по компетенциям в странах ОЭСР, но не воспроизводится в России. Например, прирост грамотности чтения и математической грамотности у россиян с высоким уровнем образования по сравнению с теми, чей уровень образования является низким, составляет соответственно 23,3 и 26,5 баллов, в то время как в среднем по странам ОЭСР этот прирост вдвое больше, и составляет 51,7 и 58,6 баллов.

Для России выявлено нетипичное влияние формального образования и грамотности чтения на вероятность быть востребованным на рынке труда. Рисунок 1 показывает эффект воздействия числа лет, потраченных на получение формального образования, а также измеренной в PIAAC грамотности в области чтения на вероятность быть вовлеченным в трудовые отношения. 
Рисунок 1. Влияние формального образования и грамотности чтения на вероятность быть востребованным на рынке труда*

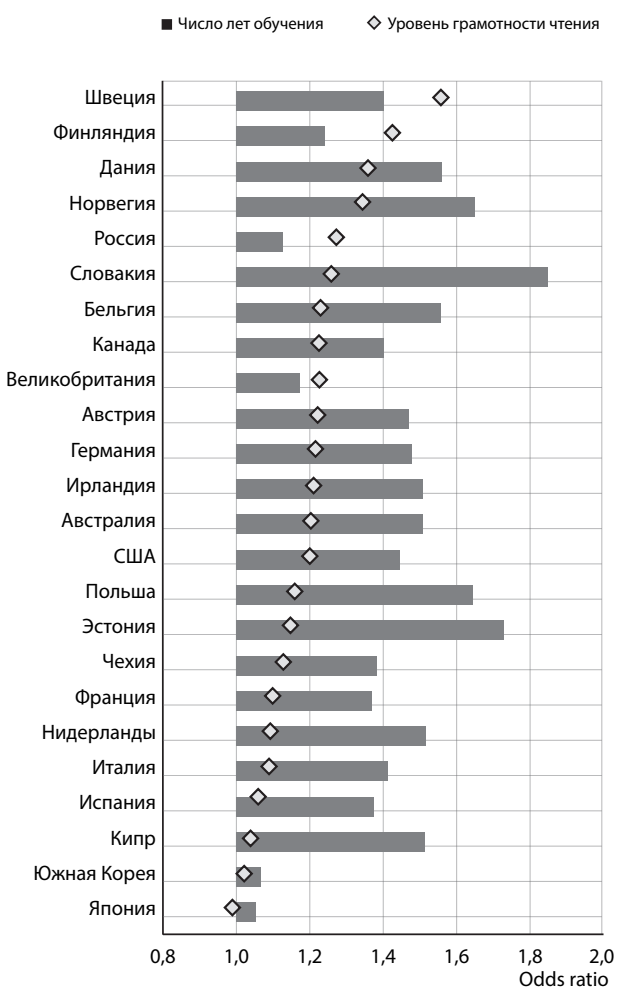

Япония

Южная Корея

Кипр

Испания

Италия

Нидерланды

Франция

Чехия

Эстония

Польша

США

Австралия

Ирландия

Германия

Австрия

Великобритания

Канада

Бельгия

Словакия

Россия

Норвегия

Дания

Финляндия

Швеция Число лет
обучения

1,054199

1,067757

1,512413

1,375557

1,412531

1,517066

1,370234

1,383013

1,727419

1,643949

1,444192

1,507716

1,508915

1,478171

1,470814

1,174316

1,401387

1,556929

1,847178

1,126008

1,650636

1,559691

1,241765

1,401361
Уровень грамотности чтения

0,98914

1,02125

1,03824

1,05935

1,089

1,09234

1,09874

1,12783

1,14723

1,15895

1,19959

1,20285

1,2103

1,21544

1,21954

1,22497

1,22615

1,22924

1,25848

1,27222

1,34361

1,3585

1,42499

1,55706

\footnotetext{
* Показатель Odds ratio, равный 1, означает статистически значимое изменение шансов наступления соответствующего события В при наступлении события А. Рисунок адаптирован и дополнен российскими данными.
}

Как следует из рисунка, практически во всех странах (за исключением Южной Кореи и Японии, для которых результаты не значимы) при увеличении лет, потраченных на получение формального образования, и при повышении уровня грамотности чтения, повышается шанс занять соответствующее место на рынке труда (хотя от страны к стране сила этой связи меняется). Россия оказалась среди тех стран, где воздействие уровня грамотности на участие в рынке труда достаточно высоко, однако при этом воздействие 
числа лет, потраченных на образование, ниже, чем для всех стран ОЭСР. В соответствии с этими результатами, в России возможность получения работы больше зависит от уровня грамотности, а не числа потраченных на образование лет. И, как итог, даже в ситуации кажущейся согласованности статусов (по формальному образованию и по доходу) возникает противоречие: с одной стороны, наличие формального диплома становится финансово более выгодным, чем обладание реальными компетенциями, но, с другой стороны, наличие реальных компетенций способствует более быстрому поиску работы.

Изучая взаимосвязь измеренной компетентности и позиции на рынке труда, мы обнаружили в нашей стране нетипичное распределение «грамотных» и «неграмотных» по разным видам занятости. Данные РIAAC позволяют сделать различную группировку по видам занятости. Мы использовали группировку из четырех элементов: высококвалифицированная работа, «белые воротнички», «синие воротнички», неквалифицированная работаотражающую базовые категории международного классификатора труда ISCO (ISCO-082004). Согласно полученным результатам, в России люди с низким уровнем измеренной грамотности часто занимаются высококвалифицированной работой: в эту группу занятости попадают $39 \%$ от числа всех низко грамотных россиян, причем эта доля заметно выше, чем в странах ОЭСР (16\%). И наоборот, в группе «высоко грамотных» доля тех, кто занимается таким трудом, заметно ниже по сравнению с другими странами: рабочие места, требующие высокой квалификации, занимают только $52 \%$ россиян из «высоко грамотной» группы, в то время как в среднем по странам ОЭСР - 76\%. Заметные различия наблюдаются также в группе «синих воротничков», к этой группе относятся $24 \%$ россиян с высоким уровнем измеренной грамотности, и 6\% высоко грамотных респондентов из стран ОЭСР. Кроме того, и в России, и в странах ОЭСР люди, имеющие низкий уровень формального образования и показавшие при этом высокие результаты тестов грамотности, чаще оказываются занятыми на малых предприятиях (численностью от 1 до 50 человек). Но при этом доли высоко грамотных, занятых на крупных и маленьких предприятиях, различаются в странах ОЭСР в 2,8 раза, а в России - в 4,9 раза. Таким образом, в России «грамотные, но необразованные» чаще, чем в странах ОЭСР, склонны выбирать небольшие компании для своей работы.

\section{Обсуждение.}

\section{Причины и следствия выявленной неконсистентности}

Эмпирические результаты вскрывают неконсистентность по нескольким показателям в сфере образования и труда. Было показано, что с ростом образовательного уровня в России, в отличие от стран ОЭСР, не всегда происходит прирост измеренной компетентности. В частности, при сравнении 
компетентности людей со средним специальным образованием и с высшим образованием, прирост грамотности статистически не значим. Обладатели среднего специального и просто среднего образования показывают результаты на уровне стран ОЭСР. В России не наблюдается прямой зависимости между уровнем образования и измеренной грамотностью, т. е. формальное образование не является надежным индикатором обладания теми или иными компетенциями, не гарантирует их освоение и передачу.

Вторым важным результатом стало, что на фоне развитых стран значительная доля (39\%) респондентов с низким уровнем измеренной грамотности в России занимает высококвалифицированные рабочие места. Однако при анализе распределения рабочих мест обладателей формальных дипломов об образовании в нашей стране мы видим картину весьма близкую к той, что наблюдается в странах ОЭСР. Иными словами, формальные дипломы не выполняют функцию эффективного индикатора образовательного уровня и/или рабочие позиции, маркированные как «высококвалифицированные», не являются таковыми в действительности.

Наконец, третий эмпирический результат демонстрирует соотношение важности измеренной грамотности и наличия формального диплома при трудоустройстве. В нашей стране оба этих фактора оказываются значимыми, но измеренная грамотность сильнее связана с шансом быть трудоустроенным по сравнению с формальным дипломом. В данном случае неконсистентность вроде бы отсутствует, однако это не совсем так. Сравнение доходов групп с высокой и низкой измеренной грамотностью было показано ранее (Кузьмина, Попов 2015). Согласно этим результатам, 25 \% представителей «высоко грамотной» группы в странах ОЭСР в среднем имеют доход в верхнем дециле, в то время как в России их доля в высоко оплачиваемой группе составляет только 4,3\%. При этом различие по уровню доходов между респондентами с низким и высоким уровнем грамотности в России значительно меньше, чем в странах ОЭСР. Если в среднем по ОЭСР доля получающих наивысший уровень зарплат в упомянутых группах отличается на $20 \%$, то в России отличие-менее чем на $4 \%$.

В предыдущих исследованиях было показано, что учащиеся и их семьи рассматривают образование, ориентируясь на снижение затрат на диплом при увеличении степени выигрыша от него (Константиновский и др. 2015: 35). Наряду с этим была продемонстрирована неизменная эффективность дипломов о высшем образовании для повышения уровня оплаты труда, которая, впрочем, с годами постоянно уменьшается (Лукьянова 2010). Это приводит к появлению значительной группы низко грамотных людей с дипломами о высшем образовании. Биографические свидетельства позволяют говорить о ситуации преобладающей значимости диплома, как о типичной:

Мы пришли к выводу, что лучше подавать документы в **, потому что [мама] была не очень уверена, что я поступлю на бюджет, а там <..> было не так дорого. Короче, я образование не воспринимала как что-то, что является моей целью в жизни (жен., 25, офис-менеджер). 
Судя по выявленной неконсистентности, эта логика получила довольно широкое распространение, причем уже в советское время:

Я решила, что поступлю хоть как. Лишь бы какое. Лишь бы высшее образование. <.. > Живем мы рядом с *** институтом, одна остановка. Поступила и закончила институт (жен., 50 лет, высшее образование, менеджер среднего звена).

Мотивация, связанная с овладением профессией, здесь подменяется внешней мотивацией, ориентированной на получение важного социального маркера - образовательного диплома. Однако на этом фоне мы не наблюдаем падения результатов формального образования на нижних его уровнях на фоне ведущих мировых стран, и этот вопрос представляет отдельный интерес. Как было показано, результаты формального образования начального и среднего уровня отличаются для тех, кто занят на малых и больших предприятиях. При этом высоко грамотные, но не имеющие высшего образования, чаще заняты на малых предприятиях. Изучение результатов качественного проекта позволило выявить и описать эту особенность с другого ракурса. Согласно материалам интервью, инструментальное отношение к образованию для самозанятых и работников малых предприятий является более характерным, чем для занятых в крупных компаниях, в корпоративном секторе:

Если бы я работал где-то на заводе, то оно [формальное образование] мне было бы необходимо, чтобы продвинуться по карьерной лестнице, скажем так. <...> Для себя не вижу смысла идти в институт (муж., 25 лет, средне-специальное образование, продавец).

Для этого сегмента наличие диплома о высшем образовании зачастую не воспринимается необходимым, тогда как приобретение новых навыков вне формального образования (например, благодаря полезным связям или самообразованию) может принести ощутимые результаты:

Смогла чего-то добиться только благодаря опыту работы. А образование - не существует такого образования нигде, никто не учится на управляющего рестораном <... >. Куда надо пойти, чтобы этому научиться? Только опыт (жен., 27, среднее образование, администратор кафе).

В корпоративном же секторе важность формального диплома остается ключевым фактором при формировании образовательных траекторий. Можно предположить, что изменение образовательных стратегий и мотивации обусловлено также изменениями требований рынка труда к типу квалификации (прежде всего - увеличением доли «сервисных» профессий, требующих немалой гибкости):

Чем сложнее я беру заказы, тем более интересные у меня потом появляются. Получая такие заказы, я развиваюсь, я же лазаю в Интернет, 
смотрю, как там правильно конструкции построить <..>. Если бы я училась в институте и была хорошей девочкой, я <..> не получила бы этого опыта, много бы чего не знала (жен., 28 лет, неоконченное среднее образование, швея, самозанятая).

Возвращаясь к тезису о неконсистентности как индикатору социальной нестабильности, важно дать оценку тому, каковы последствия выявленных неконсистентностей для социальной системы сегодня и в обозримом будущем. Исследования отечественных экономистов последних лет (Лукьянова 2010; Рощин, Рудаков 2015) отчетливо показывают, что экономическая отдача от получения диплома о высшем образовании за последние пятнадцать лет неуклонно снижается. На фоне этого мы продемонстрировали, что прирост реальных знаний при получении высшего образования в нашей стране минимален в сравнении со странами ОЭСР.

В заключение отметим, что в рамках статьи мы не решали задачи, связанные с анализом территориальных вариаций в сфере образования и занятости. В то же время особенность России заключается в очень сильном различии между столичными городами и провинцией в том, что касается развития рынка труда и возможностей применения компетенций. В этой ситуации можно предположить: региональные различия нивелируют эффект большей компетенции при найме в провинции, в небольших населенных пунктах. Это важный момент, которому, на наш взгляд, стоит уделить пристальное внимание в последующих исследованиях.

Можно высказать серьезные сомнения, что рынок труда в нашей стране и дальше будет «проглатывать» специалистов с формальным дипломом, но без достаточных профессиональных навыков. Здесь возникает точка социального напряжения, и одной из возможных мер для ее преодоления может стать создание и поддержка гибкой системы образования для взрослых, включающей возможности как для людей в корпоративном секторе, так в области малого и среднего бизнеса и для самозанятых.

\section{Выражения благодарности}

При подготовке статьи использовались материалы проекта «Межпоколенная социальная мобильность от XX века к XXI: четыре генерации российской истории», поддержанного Российским научным фондом. Грант № 14-28-00217.

\section{Список источников}

Бурдье П., Пассрон Ж.-К. (2007) Воспроизводство: элементы теории системы образования, М.: Просвещение.

Гимпельсон В.Е., Капелюшников Р. И., Карабчук Т. С., Рыжикова 3. А., Биляк Т. А. (2009) Выбор профессии: чему учились и где пригодились? Alma mater, (10): 54-67.

Карпенко М.П. (ред.) (2012) Качество высшего образования, М.: Изд-во СГУ. 
Константиновский Д. Л., Абрамова М.А., Вознесенская Е. Д., Гончарова Г.С., Костюк В. Г., Попова Е.С., Чередниченко Г. А. (2015) Новые смыслль в образовательных стратегиях молодежи: 50 лет исследования, М.: ЦСПиМ.

Кузьмина Ю.В., Попов Д.С. (2015) Функциональная грамотность взрослых и их включенность в общество в России. Социологические исследования, (7): 48-57.

Лукьянова А. Л. (2010) Отдача от образования: что показывает мета-анализ. Экономический журнал ВШЭ, (3): 326-348.

Рощин С. Ю., Рудаков В.Н. (2015) Измеряют ли стартовые заработные платы выпускников качество образования? Обзор российских и зарубежных исследований. Вопросы образования, (1): 137-181.

Селезнева Н.А. (2003) Качество высшего образования как объект системного исследования, М.: Исследовательский центр проблем качества подготовки специалистов.

Collins R. (1979) The Credential Society: An Historical Sociology of Education and Stratification, New York: Academic Press.

Hanushek E. A., Schwerdt G., Wiederhold S., Woessmann L. (2015) Returns to Skills Around the World: Evidence from PIAAC. European Economic Review, 73 (C): 103-130.

ISCO-08 (2004) Structure and Preliminary Correspondence with ISCO-88. Доступно по ссылке: https:/goo.gl/ZUBLUS (дата обращения: 30 августа 2016).

Meyer J. W. (1977) The Effects of Education as an Institution. The American Journal of Sociology, 83 (1): 55-77.

OECD (2013) OECD Skills Outlook 2013: First Results from the Survey of Adult Skills, Paris: OECD Publishing.

Powell W. W., DiMaggio P. (1991) Introduction. W. Powell, P. DiMaggio (eds.) The New Institutionalism in Organizational Analysis, Chicago, IL: University of Chicago Press: 1-38.

Wiseman A. W., Astiz F. M., Baker D. P. (2014) Comparative Education Research Framed by Neo-Institutional Theory: a Review of Diverse Approaches and Conflicting Assumptions. A Journal of Comparative and International Education, 44 (5): 688-709. 
Dmitry Popov, Anna Strelnikova

\title{
WORK, EDUCATION AND LITERACY IN RUSSIAN SOCIETY: THE PROBLEM OF INCONSISTENCY
}

\begin{abstract}
In this paper we consider the problem of inconsistency between levels of education and work in Russia. The study is based on the data from international large-scale assessments of adult competences (PIAAC), which provides us with the results of competency tests on literacy and numeracy of adults in Russia and the OECD countries. These assessment results are representative for the able-bodied population in each country at the national level. While test scores in the OECD countries show a linear correlation with the formal education levels, in Russia we observe distinctive tendencies. The analysis of the PIAAC data reveals three different inconsistencies. First, there is no linear correlation found between the assessment results and formal qualification in Russia. The increase of competence slows down or stops at the level of higher education, while at lower qualification levels there is no substantial difference between Russia and the OECD countries. The second important finding is that a significant proportion of people with low competences have high-skill jobs in Russia. At the same time, the distribution of people with certain formal qualifications on the labor market is quite similar in Russia and the OECD countries. This inconsistency was hidden for a long time due to low reliability of the most common indicator for both sociological and economic studies in Russia - the level of formal qualification. The third empirical finding demonstrates that both formal qualification and measured competences are significant in one's employment prospects. However, the difference in incomes between people with low and high competences is noticeably smaller in Russia than the OECD countries. There may be different explanations for this finding. Either employers are not interested in highly competent workers or there is a certain difference between what appear to be the same job positions in Russia and other countries. The interpretation of quantitative results in this article is also supported by interviews from a qualitative project focusing on the life trajectories.
\end{abstract}

Key words: Social inequality, education, inconsistency in education, literacy, competence-based approach

DOI: $10.17323 / 727-0634-2017-15-2-267-280$

Dmitry Popov - PhD (kandidat nauk) in Sociology, Senior Researcher at The National Research University Higher School of Economics (HSE), Moscow, Russian Federation. Email: dmitry_popov@sociolog.net

Anna Strelnikova- PhD (kandidat nauk) in Sociology, Associate Professor in Sociology at The National Research University Higher School of Economics (HSE), and Associate Professor in Sociology at GAUGN, Moscow, Russian Federation. Email: astrelnikova@hse.ru 


\section{References}

Bourdieu P., Passeron J.-C. (1990) Vosproizvodstvo: jelementy teorii sistemy obrazovanija [Reproduction in Education, Society and Culture], Moscow: Prosveshchenie.

Collins R. (1979) The Credential Society: An Historical Sociology of Education and Stratification, New York: Academic Press.

Gimpel'son V.E., Kapelyushnikov R. I., Karabchuk T. S., Ryzhikova Z. A., Bilyak T. A. (2009) Vybor professii: chemu uchilis' i gde prigodilis'? [Choosing a Profession: From Education to Job]. Alma mater, (10):54-67.

Hanushek E. A., Schwerdt G., Wiederhold S., Woessmann L. (2015) Returns to Skills Around the World: Evidence from PIAAC. European Economic Review, 73 (C): 103-130.

ISCO-08 (2004) Structure and Preliminary Correspondence with ISCO-88. Available at: https://goo.gl/ZUBLUS (accessed 30 August 2016).

Karpenko M.P. (ed.) (2012) Kachestvo vysshego obrazovaniya [The Quality of Higher Education], Moscow: Izd-vo SGU.

Konstantinovskiy D.L., Abramova M.A., Voznesenskaya E.D., Goncharova G. S., Kostyuk V. G., Popova E. S., Cherednichenko G. A. (2015) Novye smysly v obrazovatel'nykh strategiyakh molodezhi: 50 let issledovaniya [New Senses of Youth Education Strategies: the 50 Years of Researches], Moscow: TsSPiM.

Kuz'mina Yu.V., Popov D. S. (2015) Funktsional'naya gramotnost' vzroslykh i ikh vklyuchennost' v obshchestvo v Rossii [Functional Literacy of Adults and Social Inclusion]. Sotsiologicheskie issledovaniya [Sociologic Research], (7):48-57.

Luk'yanova A.L. (2010) Otdacha ot obrazovaniya: chto pokazyvaet meta-analiz [The Education Outcome: Results of Meta-Analysis]. Ekonomicheskiy zhurnal VShE [HSE Economic Journal], (3): 326-348.

Meyer J. W. (1977) The Effects of Education as an Institution. The American Journal of Sociology, 83 (1): 55-77.

OECD (2013) OECD Skills Outlook 2013: First Results from the Survey of Adult Skills, Paris: OECD Publishing.

Powell W. W., DiMaggio P. (1991) Introduction. W. Powell, P. DiMaggio (eds.) The New Institutionalism in Organizational Analysis, Chicago, IL: University of Chicago Press: 1-38.

Roshchin S. Yu., Rudakov V.N. (2015) Izmeryayut li startovye zarabotnye platy vypusknikov kachestvo obrazovaniya? Obzor rossiyskikh i zarubezhnykh issledovaniy [Are the Initial Salaries Correspond to the Quality of Education? Review of Russian and International Issues]. Voprosy obrazovaniya [Issues of Education], (1): 137-181.

Selezneva N.A. (2003) Kachestvo vysshego obrazovaniya kak ob"ekt sistemnogo issledovaniya [The Quality of Higher Education as Object for System Analysis], Moscow: Issledovatel'skiy tsentr problem kachestva podgotovki spetsialistov.

Wiseman A. W., Astiz F. M., Baker D. P. (2014) Comparative Education Research Framed by Neo-Institutional Theory: A Review of Diverse Approaches and Conflicting Assumptions. A Journal of Comparative and International Education, 44 (5): 688-709. 